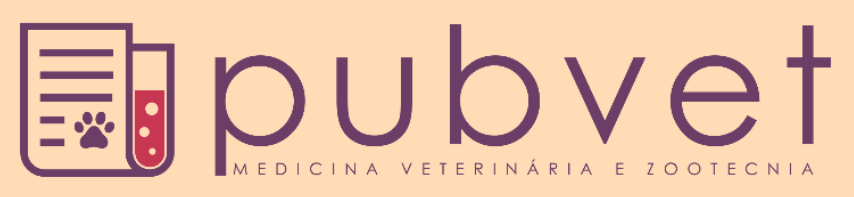

https://doi.org/10.31533/pubvet.v14n7a611.1-5

\title{
Cardiomiopatia arritmogênica do ventrículo direito em felinos: revisão
}

\author{
Maurício Andrade Bilhalva ${ }^{1^{*} \bullet}$, Adeline Bogo Madril ${ }^{1 \bullet}$, Franciéli Ribeiro da Silva ${ }^{1}$, Thaíssa \\ Gomes Pellegrin $^{1}{ }^{\circ}$, Katia Jaggi ${ }^{\circ}{ }^{\circ}$, Karmilee dos Santos Pontes ${ }^{\circ}{ }^{\circ}$, Georgina Virgínia Lavor \\ Chacon $^{2}$, Raqueli Teresinha França ${ }^{3} \odot$ (D), Paula Priscila Correia Costa ${ }^{3}{ }^{\circ}$ \\ ${ }^{I}$ Discente em medicina veterinária na Universidade Federal de Pelotas. Pelotas -RS Brasil. \\ ${ }^{2}$ Msc.,Discente em medicina veterinária na Universidade Estadual do Ceará. Fortaleza -CE Brasil. \\ ${ }^{3}$ Médica veterinária Professora na Universidade Federal de Pelotas, departamento de clínicas. Pelotas -RS Brasil. *Autor para \\ correspondência: E-mail: mauricioandradebilhalva@gmail.com
}

\begin{abstract}
Resumo. A Cardiomiopatia Arritmogênica do Ventrículo Direito (CAVD) é uma enfermidade herdada geneticamente que consiste em morte dos cardiomiócitos e substituição por tecido fibroadiposo. São raros os relatos de caso em felinos domésticos. Sinais clínicos como síncope, insuficiência cardíaca direita e arritmias são sugestivos da doença, porém, algumas pacientes podem ser assintomáticos. Exames complementares como eletrocardiograma, ecocardiograma, radiografia e análise histopatológica são importantes para o diagnóstico. O elevado risco de morte súbita torna desafiador o tratamento e controle da doença.
\end{abstract}

Palavras chave: arritmias, cardiopatia, CAVD, morte súbita

\section{Arrhythmogenic right ventricular cardiomyopathy in Felines: review}

\begin{abstract}
Arrhythmogenic Right Ventricular Cardiomyopathy is a genetically inherited disease that consists of the death of cardiomyocytes and replacement by fibroadipose tissue. Case reports in domestic cats are rare. Clinical signs such as syncope, right heart failure (CHF) and arrhythmias are suggestive of the disease, however, some patients may be asymptomatic. Complementary tests such as electrocardiogram, echocardiogram, radiography and histopathological analysis are important for the diagnosis. The high risk of sudden death makes the treatment and control of the disease challenging.
\end{abstract}

Keywords: arrhythmias, ARVC, heart disease, sudden death

\section{Miocardiopatía ventricular derecha arritmogénica en felinos: revisión}

Resumen. La miocardiopatía ventricular derecha arritmogénica (MAVD) es una enfermedad genéticamente heredada que consiste en la muerte de los cardiomiocitos y el reemplazo por tejido fibroadiposo. Los informes de casos en gatos domésticos son raros. Los signos clínicos como el síncope, la insuficiencia cardíaca derecha y las arritmias sugieren la enfermedad, sin embargo, algunos pacientes pueden ser asintomáticos. Las pruebas complementarias como el electrocardiograma, el ecocardiograma, la radiografía y el análisis histopatológico son importantes para el diagnóstico. El alto riesgo de muerte súbita dificulta el tratamiento y el control de la enfermedad.

Palabras clave: arritmias, enfermedad cardíaca, MAVD, muerte súbita

\section{Introdução}

A cardiomiopatia arritmogênica do ventrículo direito (CAVD) é uma enfermidade primária do miocárdio que ocorre pela morte de cardiomiócitos seguida pela substituição de tecido fibroso e adiposo, 
gerando uma atrofia progressiva no ventrículo afetado (Backschat et al., 2016; Harvey et al., 2005). Essa modificação tecidual promove o surgimento de arritmias ventriculares, pois detém a continuidade elétrica das fibras miocárdicas, fragmentando a despolarização e produzindo potenciais ventriculares retardados (Larsson \& Chamas, 2011). Embora se trate de uma cardiopatia rara em felinos, a CAVD pode afetar algumas raças de cães, especialmente o Boxer e, principalmente, seres humanos, indivíduos mais comumente afetados pela doença e vítimas de morte súbita oriunda dessa enfermidade (Backschat et al., 2016; Larsson \& Chamas, 2011). Ainda que sua ocorrência não seja comum em felinos, seu estudo é importante, tendo em vista que o distúrbio ainda é pouco elucidado pela medicina veterinária (Fox et al., 2000).

A doença foi descrita na espécie felina pela primeira vez por Fox et al. (2000), em um estudo retrospectivo, no qual doze gatos haviam sido diagnosticados com a CADV. No Reino Unido, foram relatados por Harvey et al. (2005) os dois primeiros casos em território britânico. Em 2009, na Itália, foi descrito um caso de comprometimento importante de ventrículo esquerdo de um paciente felino com a doença (Ciaramella et al., 2009). No Brasil, apenas em 2016, ocorreu o primeiro relato de ocorrência de cardiomiopatia arritmogênica de ventrículo direito em um felino (Backschat et al., 2016).

O objetivo dessa revisão de literatura centra-se em fazer um sucinto apanhado das informações já registradas na literatura a respeito da ocorrência da CAVD em felinos domésticos.

\section{Etiopatogenia}

A etiopatogenia desta enfermidade em felinos ainda permanece incerta, por causa da baixa incidência de cardiomiopatia arritmogênica do ventrículo direito na espécie em questão (Backschat et al., 2016). Segundo Harvey et al. (2005), a apoptose das células, processos inflamatórios, atrofia associada a fatores genéticos e fatores imunomediados, são apontados como possíveis mecanismos patogênicos. Contudo, nenhuma predisposição por idade ou raça foi identificada (Ciaramella et al., 2009).

Trata-se de uma doença miocárdica primária familiar que prevalece nos cães da raça boxer. Nesses casos, a enfermidade é descrita como herdada com característica autossômica dominante, como ocorre em seres humanos. No entanto, nem as proteínas codificadas defeituosas, tampouco os defeitos genéticos específicos, foram identificadas com exatidão nos felinos (Corrado et al., 2000; Fox et al., 2000). Em estudos com seres humanos, a CAVD é considerada causa de morte súbita em jovens e atletas, e se sabe que ocorre por causa de mutações em genes codificadores das proteínas desmossomais. A disfunção é tida como geneticamente estabelecida e causadora de distrofia miocárdica (Basso et al., $\underline{2009)}$.

\section{Sinais clínicos}

O principal sinal clínico associado à patologia é a presença de complexos prematuros ventriculares (VPC) originados no ventrículo direito. Além disso, é possível observar a presença de outras arritmias como taquicardia ventricular, fibrilação atrial, taquicardia supraventricular e ventricular (Harvey et al., 2005). Esse mesmo estudo descreveu o primeiro relato de CAVD em felinos domésticos na Europa. Neste trabalho, avaliaram-se dois animais, nos quais se observaram sinais clínicos de insuficiência cardíaca congestiva (ICC) do lado direito, com taquipneia, pulsação da veia jugular, hepatomegalia e ascite, resultados semelhantes aos sinais descritos por Fox et al. (2000). Já a síncope ocorreu em um dos dois gatos. $\mathrm{O}$ exame de radiografias torácicas demonstrou cardiomegalia.

Em um estudo realizado por Fox et al. (2000), avaliaram-se 12 gatos domésticos com cardiomiopatia arritmogênica do ventrículo direito. Oito deles manifestaram ICC do lado direito associado a taquipnéia, distensão da veia jugular, derrame abdominal ou hepatoesplenomegalia e a síncope ocorreu em 1 animal. Em oito gatos foi possível identificar sopros cardíacos pansistólicos consistentes com regurgitação tricúspide. Os outros 4 animais restantes apresentaram letargia e anorexia, mas não apresentaram evidências de ICC.

\section{Diagnóstico}

O diagnóstico da CAVD é baseado nos sinais clínicos e em exames complementares, tais como radiografia torácica, ecocardiograma, eletrocardiograma convencional e ambulatorial (Holter) e 
investigação histopatológica (Fox et al., 2000; Ciaramella et al., 2009; Harvey et al., 2006). Os principais diagnósticos diferenciais incluem anomalias congênitas que cursam com alterações na câmara cardíaca direita, como ocorre nos casos de displasia de tricúspide e anomalia de Uhl (Ciaramella et al., 2009).

No ecocardiograma, os principais achados que indicam a enfermidade incluem o aumento das câmaras cardíacas direitas, diminuição da espessura da parede do ventrículo direito resultante da dilatação, insuficiência de válvula tricúspide e aneurismas. Na radiografia, observa-se principalmente o aumento da silhueta cardíaca em decorrência da dilatação do átrio e ventrículo direito, além de efusão pleural (Meurs, 2005). O eletrocardiograma também serve para verificar algumas alterações, incluindo diversas arritmias, como taquicardia ventricular, fibrilação atrial, taquicardia supraventricular, complexos ventriculares prematuros, bloqueio do ramo direito e esquerdo e bloqueio atrioventricular de primeiro grau (Fox et al., 2000; Ciaramella et al., 2009; Harvey et al., 2006). Os complexos ventriculares prematuros de origem ventricular direita são os mais frequentes e podem ocorrer mesmo em animais assintomáticos (

A análise histopatológica do miocárdio (Figuras 1A e 1B) auxilia muito no diagnóstico de CAVD, porém é uma modalidade diagnóstica difícil de se realizar in vivo. O laudo revela infiltração fibroadiposa no miocárdio, especialmente na parede do ventrículo direito e resulta em atrofia miocárdica de forma focal ou difusa. Além disso, pode ocorrer achados de miocardite focal ou multifocal do ventrículo direito. Os infiltrados inflamatórios são compostos principalmente de linfócitos $\mathrm{T}$ relacionados à morte celular dos cardiomiócitos seguida de reposição fibrosa (Fox et al., 2000; Harvey et al., 2006). Na necropsia o achado mais evidente corresponde ao aumento da cavidade do ventrículo e átrio direitos e o estreitamento da parede ventricular direita, assim como aneurismas, conforme se identifica na figura 2 (Fox et al., 2000).
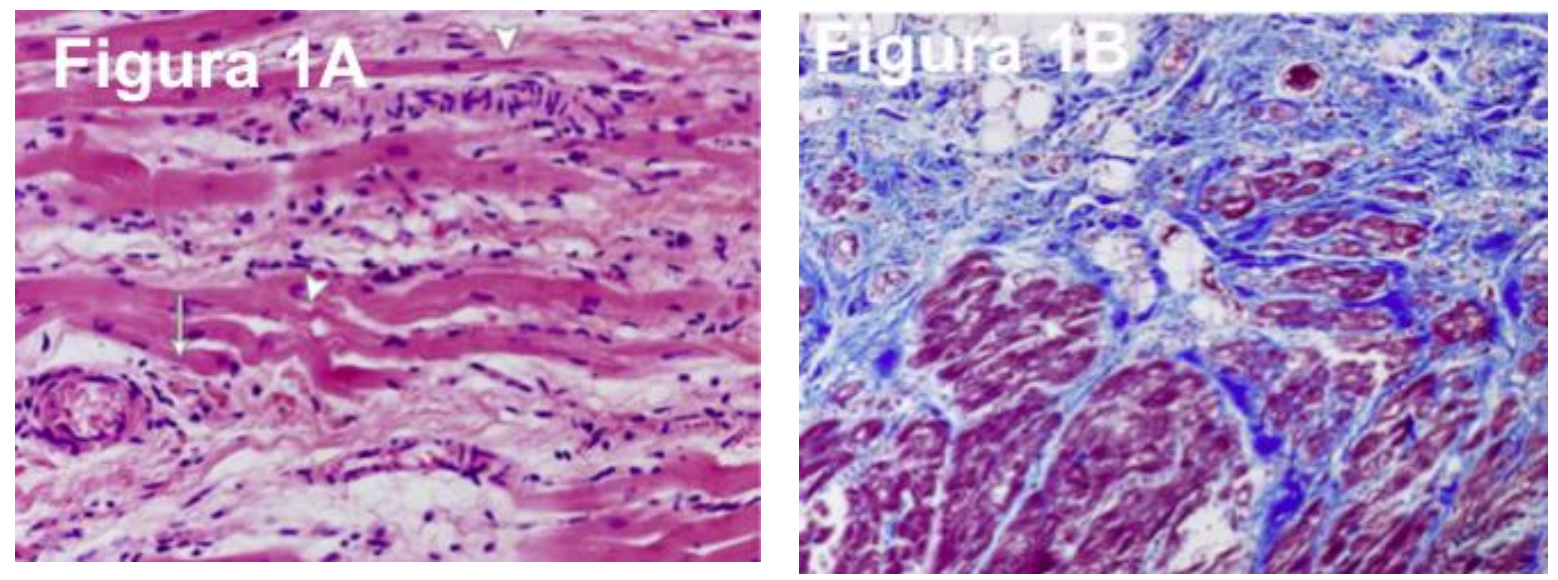

Figura 1A. Histologia do miocárdio do ventrículo direito, parede livre ântero-lateral. Há ondulação e alongamento dos miócitos, o que sugere necrose focal demonstrada pelas pontas de seta. Algumas células inflamatórias redondas também são visíveis a partir das setas. Figura 1B. Histologia do miocárdio do ventrículo direito, parede livre ânterolateral. É possível visualizar a substituição do miocárdio por tecido fibroso (cor azul) e a presença de alguns adipócitos. Fonte: Ciaramella et al. (2009).

\section{Tratamento}

Assim como ocorre em humanos, devido ao elevado risco de morte súbita dos pacientes acometidos pela enfermidade, ainda não se estabeleceram diretrizes precisas para determinar quais são os pacientes que precisam ser tratados e qual é a melhor abordagem de gerenciamento (Corrado et al., 2000). Fox et al. (2000) recomendam o uso de diuréticos, inotropicos positivos e inibidores da ECA.

Nos casos em cães da raça boxer, os mais acometidos pela enfermidade, recomenda-se o uso de agentes antiarrítmicos, a menos que haja indicadores de disfunção sistêmica e ICC. O tratamento antiarrítmico tende a diminuir a frequência e a complexidade do VPC, bem como o número de episódios de síncope. No entanto, não há registros de que esse recurso terapêutico afete a sobrevida a longo prazo ou o risco de morte súbita. Além disso, o tratamento pode causar efeitos colaterais importantes, uma vez que os agentes antiarrítmicos podem ter um efeito pró-arrítmico ( 


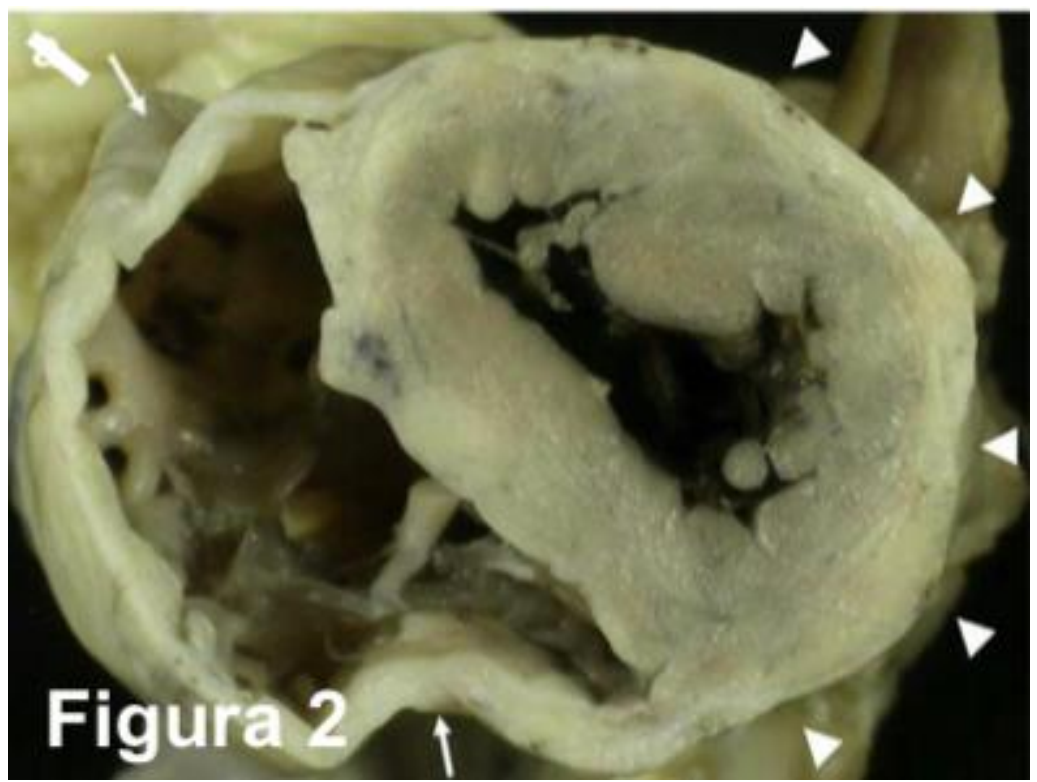

Figura 2. Corte cardíaco transversal post-mortem realizado no nível ventricular médio O ventrículo direito está bastante dilatado. É possível observar o afinamento difuso da parede livre do ventrículo direito. Evidencia-se a presença de aneurismas ântero-infundibulares e inferiores apontados através das setas. Ao longo de toda a circunferência da parede livre do ventrículo esquerdo, é evidente uma banda subepicárdica esbranquiçada (pontas de seta). Fonte: Ciaramella et al. (2009).

Em humanos, recomenda-se submeter os pacientes a acompanhamento cardíaco regularmente, incluir histórico médico, ECG basal, monitoramento com uso de Holter, teste ergométrico e ecocardiograma para identificar de forma precoce sintomas de alerta, início ou agravamento de anormalidades morfofuncionais do ventrículo direito e arritmias ventriculares. Além disso, algumas evidências sugerem que pacientes assintomáticos ou sem alterações genéticas não precisam de tratamento profilático (Basso et al., 2009).

\section{Prognóstico}

Segundo a literatura, a forma como a doença ocorre pode variar de acordo com cada paciente. No entanto, o prognóstico varia de reservado a desfavorável. Os estudos que relatam a doença em felinos domésticos apresentam um alto grau de mortalidade nesta espécie. Muitos pacientes apresentam mortes súbitas (Fox et al., 2000; Ciaramella et al., 2009; Harvey et al., 2005). A insuficiência cardíaca direita progressiva mostrou-se uma importante causa de morte em pacientes felinos (Fox et al., 2000).

Ainda há muito pouco conhecimento a respeito dessa doença nos felinos, uma vez que não se trata de uma doença comum e ainda se tem um número baixo de relatos nessa espécie. No entanto, a exemplo de cães da raça Boxer, que são animais mais frequentemente acometidos, alguns pacientes podem passar anos assintomáticos, enquanto outros são controlados facilmente com antiarrítmicos. Entretanto, o risco de morte súbita está presente (Silva et al., 2014).

\section{Avaliação clínica}

A doença representa um grande desafio na clínica de felinos porque além de ser rara, é uma enfermidade com sintomas que podem se confundir com outras doenças. É essencial que o médico veterinário saiba da possibilidade da ocorrência desse distúrbio no momento de levantar as variáveis diagnósticas quando encontrar tais sintomas. Em alguns casos a constatação da doença ocorre apenas após a morte do paciente seguida pela realização da análise histopatológica (Backschat et al., 2016).

A avaliação das arritmias de origem ventricular direita, em decorrência da alteração histológica envolvida na patogenia da enfermidade é fundamental no acompanhamento clínico do paciente. Salienta-se que em casos de suspeita de CAVD em pacientes felinos seja realizado não apenas o ECG 
de rotina, mas também o sistema Holter para monitorar o animal por 24 horas para detecção de indícios mais sólidos da presença da doença (Backschat et al., 2016).

\section{Considerações finais}

A cardiomiopatia arritmogênica do ventrículo direito (CAVD) é uma doença miocárdica primária incomum, cuja patogênese ainda não está claramente estabelecida. Esta enfermidade pode oferecer graves riscos, embora raros sejam os relatos envolvendo animais da espécie felina. Devido a sua baixa prevalência nos felinos domésticos, seu diagnóstico muitas vezes é difícil e um tratamento eficiente não é estabelecido. Em vista disso, é importante continuar realizando pesquisas sobre o assunto para que assim seja possível estabelecer formas mais concisas de administração da enfermidade, visto que o diagnóstico precoce promove um melhor tratamento, controle e qualidade de vida dos animais acometidos.

\section{Referências bibliográficas}

Backschat, P. S., Goldfeder, G. T., Ampuero, F., Lacerda, A. M. D., \& Larsson, M. H. M. (2016). Cardiomiopatia arritmogênica do ventrículo direito em felino: relato de caso. Arquivo Brasileiro de Medicina Veterinaria e Zootecnia, 68(5), 1112-1116. https://doi.org/10.1590/1678-4162-8616

Basso, C., Corrado, D., Marcus, F. I., Nava, A., \& Thiene, G. (2009). Arrhythmogenic right ventricular cardiomyopathy. The Lancet, 373(9671), 1289-1300. https://doi.org/10.1056/NEJMc1701400

Ciaramella, P., Basso, C., Di Loria, A., \& Piantedosi, D. (2009). Arrhythmogenic right ventricular cardiomyopathy associated with severe left ventricular involvement in a cat. Journal of Veterinary Cardiology, 11(1), 41-45. https://doi.org/10.1016/j.jvc.2009.02.007

Corrado, D., Basso, C., \& Thiene, G. (2000). Arrhythmogenic right ventricular cardiomyopathy: diagnosis, prognosis, and treatment. Heart, 83(5), 588-595. https://doi.org/10.1136/heart.83.5.588

Fox, P. R., Maron, B. J., Basso, C., Liu, S.-K., \& Thiene, G. (2000). Spontaneously occurring arrhythmogenic right ventricular cardiomyopathy in the domestic cat: a new animal model similar to the human disease. Circulation, 102(15), 1863-1870. https://doi.org/10.1161/01.CIR.102.15.1863

Hariu, C. D., \& Carpenter, D. (2010). Arrhythmogenic right ventricular cardiomyopathy in boxers. Compendium Continuig Education for Veterinarians, 32, E3. https://doi.org/10.1016/j.jelectrocard.2007.03.192

Harvey, A. M., Battersby, I. A., Faena, M., Fews, D., Darke, P. G. G., \& Ferasin, L. (2005). Arrhythmogenic right ventricular cardiomyopathy in two cats. Journal of Small Animal Practice, 46(3), 151-156.

Larsson, M. H. M. A., \& Chamas, P. P. C. (2011). Cardiomiopatia arritmogênica do ventrículo direito. Vets Today, 7.

Meurs, K. M. (2005). Inherited Heart Disease: Diagnosis and Screening. Proceeding of the North American Veterinary Conference. Orlando, Florida, 8-12.

Silva, L. A. P., Contieri, M. B., \& Ferreira, F. S. (2014). Cardiomiopatia arritmogênica do ventrículo direito do boxer-revisãode literatura. Revista Científica de Medicina Veterinária, 12(40), 128-138.

Recebido: 10 de fevereiro, 2020.

Aprovado: 16 de março, 2020.

Disponível online: 4 de agosto, 2020.

Licenciamento: Este artigo é publicado na modalidade Acesso Aberto sob a licença Creative Commons Atribuição 4.0 (CC-BY 4.0), a qual permite uso irrestrito, distribuição, reprodução em qualquer meio, desde que o autor e a fonte sejam devidamente creditados. 